\title{
Balloon Catheter Nominal Outer Diameter
}

National Cancer Institute

\section{Source}

National Cancer Institute. Balloon Catheter Nominal Outer Diameter. NCI Thesaurus.

Code C150161.

The nominal measure of the outer diameter of the the balloon. 